\title{
On the Incorporation of Trivalent Rare Earth Ions in II-VI Semiconductor Nanocrystals
}

\author{
Ageeth A. Bol,* Rick van Beek, and Andries Meijerink \\ Utrecht University, Debye Institute, Physics and Chemistry of Condensed Matter, \\ P.O. Box 80 000, 3508 TA Utrecht, The Netherlands
}

Received J uly 20, 2001. Revised Manuscript Received November 6, 2001

\begin{abstract}
Nanocrystalline ZnS and CdS samples have been synthesized in the presence of $\mathrm{Eu}^{3+}$ and $\mathrm{Tb}^{3+}$ using various techniques (precipitation in water, methanol, or toluene and inverse micelle techniques) which have been reported to yield ZnS or CdS nanoparticles doped with luminescent rare earth ions. Nanocrystalline particles with a typical diameter of $4 \mathrm{~nm}$ were formed. In some cases, particles were heated (up to $800^{\circ} \mathrm{C}$ ) which resulted in an increase of the particle diameter (>20 nm). To study the incorporation of rare earth ions in the particles, luminescence spectra have been measured. U pon excitation in the semi conductor host lattice, no emission or weak emission is observed from the rare earth ions. The excitation spectra of the characteristic rare earth emissions show excitation lines corresponding to intraconfigurational $4 \mathrm{f}^{\mathrm{n}}-4 \mathrm{f}^{\mathrm{n}}$ transitions of the rare earth ions but not the semiconductor host lattice excitation band. The absence of a host lattice excitation band indicates that with the presently used synthesis techniques the rare earth ions are not incorporated in the nanocrystalline semiconductor particles but are probably adsorbed at the surface.
\end{abstract}

\section{Introduction}

Nanocrystalline semiconductors have been the subjects of numerous investigations in the past two decades. If the semiconductor particles become smaller than the Bohr radius of the exciton, so-called quantum size effects occur ${ }^{1-4}$ As a result of these quantum size effects, the band gap of the semiconductor increases and at the edges of the valence and conduction band discrete energy levels occur. These quantum size effects have stimulated great interest in both basic and applied research.

In 1994, Bhargava reported on remarkable optical properties of doped semiconductor nanoparticles. ${ }^{5}$ It was argued that the lifetime of the $\mathrm{Mn}^{2+}$ emission in nanocrystalline ZnS was shortened from milliseconds to nanoseconds due to quantum size effects. In addition, the luminescence quantum efficiency was reported to increase with decreasing particle size. However, it was later shown that lifetime shortening in nanocrystalline $\mathrm{ZnS} / \mathrm{Mn}^{2+}$ does not occur. ${ }^{6,7}$ The $\mathrm{Mn}^{2+}$ emission of nanocrystalline $\mathrm{ZnS} / \mathrm{Mn}^{2+}$ has a "normal" millisecond lifetime.

Despite the absence of lifetime shortening, numerous papers on the luminescence of semiconductor nanopar-

* To whom correspondence should be addressed. Present address: Philips Research, Prof. Holstlaan 4 (WA11), 5656 AA Eindhoven, The Netherlands. Tel: (+31) 40 2742271. Fax: (+31) 40 2744282. E-mail: ageeth.bol @philips.com.

(1) Rossetti, R.; Hull, R.; Gibson, J . M.; Brus, L. E. J . Chem. Phys. 1985, 82, 552.

(2) Brus L. J . Phys. Chem. 1986, 90, 2555.

(3) Henglein A. Chem. Rev. 1989, 89, 1861.

(4) Wang, Y.; Herron, N. J. Phys. Chem. 1991, 95, 525.

(5) Bhargava, R. N.; Gallagher, D. Phys. Rev. Lett. 1994, 72, 416.

(6) Murase, N.; J agannathan, R.; Kanematsu, Y.; Watanabe, M.; Kurita, A.; Hirata, K.; Yazawa, T.; Kushida, T. J . Phys. Chem. B 1999, 103, 754.

(7) Bol, A. A.; Meijerink, A. Phys. Rev. B 1998, 58, R15997. ticles doped with $3 d$ transition metal (TM) ions or rare earth (RE) ions have appeared and in various articles it was concluded that both the TM- and RE-doped ZnS nanocrystals "form a new class of luminescent materials". $5,8,9$ This report focuses on RE-doped semiconductor nanoparticles. The literature has reports on both oxidic insulators and II-VI semiconductor nanoparticles doped with RE ions. From the papers on nanocrystalline REdoped oxides, it is clear that it is very well possible to incorporate $\mathrm{RE}$ ions in oxidic nanoparticles such as $\mathrm{Y}_{2} \mathrm{O}_{3},{ }^{10-12} \mathrm{YVO}_{4},{ }^{13}$ and $\mathrm{Gd}_{2} \mathrm{O}_{3},{ }^{14}$ due to the very similar chemical properties of all rare earth ions. Quantum size effects are not expected in these oxides since the Bohr radius of the exciton in insulating oxides, like $\mathrm{Y}_{2} \mathrm{O}_{3}$, $\mathrm{YVO}_{4}$, and $\mathrm{Gd}_{2} \mathrm{O}_{3}$, is very small. The exciton Bohr radius of II-VI semiconductors is larger (e.g., $2.5 \mathrm{~nm}$ for CdS) resulting in pronounced quantum confinement effects for nanoparticles of about $2.5 \mathrm{~nm}$ and smaller. Therefore, a possible influence of quantum size effects on the luminescence properties of RE ions is only expected in II-VI semiconductor nanocrystals.

A number of papers reported on the luminescence of nanocrystalline $\mathrm{II}-\mathrm{VI}$ semiconductors doped with $\mathrm{Tb}^{3+},{ }^{15-17} \mathrm{Sm}^{2+},{ }^{18} \mathrm{Eu}^{3+} / \mathrm{Eu}^{2+}, 9,19,20$ or $\mathrm{Er}^{21}$ Papers on

(8) Yang, P.; Lü, M.; Xü, D.; Yuan, D.; Zhou, G.; Chem. Phys. Lett. 2001, 336, 76.

(9) Xu, S. J .; Chua, S. J .; Liu, B.; Gan, L. M.; Chew, C. H.; Xu, Q. Q. Appl. Phys. Lett. 1998, 73, 478.

(10) Ye, T.; Guiwen, Z.; Weiping, Z.; Shangda, X. Mater. Res. Bull. 1997, 32, 501 .

(11) Goldburt, E. T.; Kulkarni, B.; Bhargava, R. N.; Taylor, J .; Libera, M. J . Lumin. 1997, 72-74, 190.

(12) Soo, Y. L.; Huang, S. W.; Ming, Z. H.; Kao, Y. H.; Smith, G. C.; Goldburt, E.; Hodel, R.; Kulkarni, B.; Veliadis, J . V. D.; Bhargava, R. N. J . Appl. Phys. 1998, 83, 5404.

(13) Riwotzki, K.; Haase, M. J. Phys. Chem. B 1998, 102, 10129.

(14) Sun, L.; Yao, J .; Liu, C.; Liao, C.; Yan, C. J . Lumin. 2000, 8789,447 
the luminescence of nanocrystalline $\mathrm{ZnS} / \mathrm{Tb}^{3+}{ }^{15-17}$ reported emission spectra characteristic for ${ }^{5} \mathrm{D}_{4}-{ }^{7} \mathrm{~F}_{\mathrm{n}}$ transitions of the $\mathrm{Tb}^{3+}$ ion. In addition, a lifetime shortening from milliseconds to nanoseconds was reported for these nanoparticles. ${ }^{15}$ An organometallic synthesis route, ${ }^{15}$ a common precipitation technique, ${ }^{16}$ and block copolymer nanoreactors ${ }^{17}$ were used to synthesize the $\mathrm{ZnS} / \mathrm{Tb}^{3+}$ nanoparticles.

The optical properties of nanocrystalline ZnS/Sm were reported in ref 18. Nanocrystalline ZnS/Sm was prepared by using a precipitation method. The broad emission and excitation bands were tentatively assigned to the transitions between the $4 \mathrm{f}^{6}$ and $4 \mathrm{f}^{5} 5 \mathrm{~d}$ states of $\mathrm{Sm}^{2+}$ and also to the transitions of excitons bound to $\mathrm{Sm}^{2+}$.

Nanocrystalline samples of ZnS/Eu or CdS/Eu were prepared by using common precipitation methods, 17,19 a microemulsion technique, ${ }^{9}$ and sol-gel processing. ${ }^{20}$ U pon host lattice excitation, either a broad band emissi on band around $500 \mathrm{~nm}^{9,19}$ or sharp $\mathrm{Eu}^{3+}$ lines ${ }^{19}$ were observed.

An important issue that needs to be addressed is if the RE ions are really being incorporated in nanocrystalline $\mathrm{ZnS}$ or $\mathrm{CdS}$. The ionic radii of RE ions are much larger than that for $\mathrm{Zn}^{2+}$ (viz., $\mathrm{Eu}^{3+}=0.95$ and $\mathrm{Zn}^{2+}=$ $0.75 \AA^{23}$ ). If one assumes that the RE ion is incorporated on a $\mathrm{Zn}^{2+}$ lattice site, like $\mathrm{Mn}^{2+}$ ions in nanocrystall ine $\mathrm{ZnS} / \mathrm{Mn},{ }^{24}$ the $\mathrm{ZnS}$ host lattice has to deform local ly. In addition, the $3+$ charge of a RE ion on a $2+$ site has to be compensated for somewhere in the host lattice. Finally, the differences in chemical properties between $\mathrm{Zn}^{2+}$ and $\mathrm{RE}^{3+}$ will not favor substitution of $\mathrm{Zn}^{2+}$ by $\mathrm{RE}^{3+}$. Still, it is known that RE ions can be incorporated in bulk II-VI semiconductors. ${ }^{25} \mathrm{Efficient} \mathrm{luminescence}$ from intraconfigurational $4 f^{n}-4 f^{n}$ transitions has been observed for RE ions in bulk ZnS upon excitation over the band gap. 26,27,28 However, high temperatures (900$1200{ }^{\circ} \mathrm{C}^{25}$ ) are needed to accomplish this. The reported synthesis conditions for the preparation of RE-doped nanocrystalline semiconductors are very mild compared to the conditions used for bulk RE-doped semiconductors. Therefore, it is not evident that RE ions are incorporated in ZnS or CdS nanoparticles.

(15) Bhargava, R. N. J . Lumin. 1996, 70, 85. 90.

(16) Kane, R. S.; Cohen, R. E.; Silbey, R. Chem. Mater. 1999, 11,

(17) Ihara, M.; I garashi, T.; Kusunoki, T.; Ohno, K.J . Electrochem. Soc. 2000, 147, 2355.

(18) Kushida, T.; Kurita, A.; Watanabe, M.; Kanematsu, Y. J. Lumin. 2000, 87-89, 466

(19) Chen, W.; Malm, J -. O.; Zwiller, V.; Huang, Y.; Liu, S.; Wallenberg, R.; Bovin, J -. O.; Samuelson, L. Phys. Rev. B 2000, 61, 11021.

(20) Morita, M.; Rau, D.; Fujii, H.; Minami, Y.; Murakami, S.; Baba, M.; Yoshita, M.; Ákiyama, H. J . Lumin. 2000, 87-89, 478. 65

(21) Schmidt, T.; Müller, G.; Spanhel, L. Chem. Mater. 1998, 10,

(22) Gallagher, D.; Heady, W. E.; Racz, J . M.; Bhargava, R. N. J . Mater. Res. 1995, 10, 870.

(23) Handbook of Chemistry and Physics, 74th ed.; Lide, D. R., Ed.; CRC Press: Boca Raton, 1993.

(24) Soo, Y. L.; Ming, Z. H.; Huang, S. W.; Kao, Y. H.; Bhargava, R. N.; Gallagher, D. Phys. Rev. B 1994, 50, 7602.

(25) Phosphor Handbook; Yen, W. M., Shionoya, S., Eds.; CRC Press: Boca Raton, 1999.

(26) Hommel, D.; Hartmann, H.; Godlewski, M.; Langer, J. M.; Stapor, A. In RareEarth Spectroscopies; J ezowska-Trzebiatowska, B. Legendziewicz, J ., Strek, W., Eds.; World Scientific: Singapore, 1985; p 643.

(27) Zhang, H.; Shen, Y.J . Lumin. 1988, 40-41, 401.

(28) Stapor, A.; Godlewski, M.; Przybylinska, H.; Hommel, D. J . Lumin. 1988, 40-41, 625.
A few papers reported on the luminescence of RE ions adsorbed at the nanocrystal line semiconductor surface ${ }^{29}$ or nanocrystalline semiconductors adsorbed on REcontaining species. ${ }^{30,31}$ The emission spectra for these substances are very similar to the spectra reported for nanocrystalline ZnS/RE or CdS/RE. Therefore, the RE ions in the latter compounds may be located at the surface of the nanoparticle and not in the host lattice. Note that a similar discussion is going on about the incorporation of $\mathrm{Mn}^{2+}$ in nanocrystalline $\mathrm{ZnS}$ even though $\mathrm{Mn}^{2+}$ has the same charge as $\mathrm{Zn}^{2+}$ and the size and chemical properties of these ions are similar. Also for $\mathrm{ZnS} / \mathrm{Mn}^{2+}$, there is evidence that a part of the $\mathrm{Mn}^{2+}$ ions is not incorporated in the ZnS nanocrystals but is at surface sites. ${ }^{32-36}$

In this report, the incorporation of RE ions in nanocrystalline ZnS and CdS is investigated in a systematic way. Old and new synthesis methods are used and compared. Besides room-temperature preparation methods, postsynthesis heat treatment (up to $800{ }^{\circ} \mathrm{C}$ ) was done to promote incorporation of RE ions in ZnS or $\mathrm{CdS}$ nanoparticles. Even ion implantation was attempted as a method to incorporate RE ions in semiconductor nanoparticles. From careful luminescence studies, it is concluded that with the preparation techniques applied it is not possible to incorporate RE ions in nanocrystalline semiconductors.

\section{Experimental Section}

2.1. Synthesis of Nanocrystalline ZnS/E $u$ and $C d S / E ~ u$. For the synthesis of nanocrystalline $\mathrm{ZnS} / \mathrm{Eu}$ or $\mathrm{CdS} / \mathrm{Eu}$, a precipitation method and a microemulsion method were used. After the synthesis, some samples were subjected to a heat or hydrothermal treatment. The precipitation method that was used for the synthesis of nanocrystalline ZnS/Eu or CdS/Eu is similar to the method described by $\mathrm{Yu}^{37}$ for the synthesis of nanocrystalline $\mathrm{ZnS} / \mathrm{Mn}^{2+}$. A portion of $10.2 \mathrm{~g}$ of $\mathrm{Na}\left(\mathrm{PO}_{3}\right)_{\mathrm{n}}(\mathrm{PP})$ (Aldrich) was dissolved in distilled water. A portion of $10 \mathrm{~mL}$ of $1 \mathrm{M} \mathrm{Zn}\left(\mathrm{CH}_{3} \mathrm{COO}\right)_{2} \cdot 6 \mathrm{H}_{2} \mathrm{O}$ (for $\mathrm{ZnS} / \mathrm{Eu}$ ) or $1 \mathrm{M} \mathrm{Cd}\left(\mathrm{ClO}_{4}\right)_{2}$. $6 \mathrm{H}_{2} \mathrm{O}$ (for $\mathrm{CdS} / \mathrm{Eu}$ ) and $10 \mathrm{~mL}$ of $0.01 \mathrm{M} \mathrm{EuCl}_{3} \cdot \mathrm{xH}_{2} \mathrm{O}$ were added to this solution. Sometimes, $10 \mathrm{~mL}$ of a $0.02 \mathrm{M} \mathrm{LiCl}$ solution was added since $\mathrm{Li}^{+}$on a $\mathrm{Zn}^{2+}$ site can serve as charge compensator for $\mathrm{RE}^{3+}$ ions on $\mathrm{Zn}^{2+}$ sites. After the solution was stirred for $10 \mathrm{~min}, 10 \mathrm{~mL}$ of $1 \mathrm{M} \mathrm{Na} \mathrm{N}_{2} \mathrm{~S} \cdot 9 \mathrm{H}_{2} \mathrm{O}$ was injected. Immediately, a white suspension is formed. After the reaction, the total volume was $100 \mathrm{~mL}$. The suspension was centrifuged and washed with distilled water and ethanol. The nanoparticles were dried in a vacuum.

The microemulsion method used to prepare nanocrystalline $\mathrm{ZnS} / \mathrm{E} \mathrm{u}$ or $\mathrm{CdS} / \mathrm{E} \mathrm{u}$ is very similar to the method described by $\mathrm{Xu}$ et al. $^{9}$ for the preparation of $\mathrm{ZnS} / \mathrm{Eu}^{3+}$. As surfactant, a 2:1 mixture of poly(oxyethylene) ${ }_{5}$ nonylphenol (Empilan NP5) and poly(oxyethylene), ${ }_{9}$ nonylphenol (Empilan NP-9) of Al-

(29) Sun, L.; Yan, C.; Liu, C.; Liao, C.; Li, D.; Yu, I. J . Alloys Compd. 1998, 257-277, 234

(30) Hayakawa, T.; Selvan, S. T.; Nogami, M.J . Lumin. 2000, 87$89,532$.

(31) Reisfeld, R.; Graft, M.; Saridarov, T.; Panczer, G.; Zelner, M. Mater. Lett. 2000, 45, 154.

(32) Yu, I.; Isobe, T.; Senna, M.; Takahashi, S.; Mater. Sci. Eng. 1996, B38, 177.

(33) Borse, P. H.; Srinivas, D.; Shinde, R. F.; Date, S. K.; Vogel, W.; Kulkarni, S. K. Phys. Rev. B 1999, 60, 8659.

(34) I garashi, T.; I sobe, T.; Senna, M. Phys. Rev. B 1997, 56, 6444.

(35) Kennedy, T. A.; Glaser, E. R.; Klein, P. B.; Bhargava, R. N.; Phys. Rev. B 1995, 52, R14356.

(36) Chen, W.; Sammynaiken, R.; Huang, Y.; Malm, J .-O.; Wallenberg, R.; Bovin, J .-O.; Zwiller, V.; Kotov, N. A. J . Appl. Phys. 2001 89, 1120 . 373.

(37) Yu, I.; Isobe T.; Senna, M. J . Phys. Chem. Solids 1996, 57, 
bright $\&$ Wilson was used. Two solutions were prepared. The first one contained $75.6 \mathrm{~g}$ of petroleum ether (bp $60-80{ }^{\circ} \mathrm{C}$, Fluka), $32.4 \mathrm{~g}$ of surfactant, and $92 \mathrm{~g}$ of a $0.1 \mathrm{M} \mathrm{ZnCl} / 2 / 0.01 \mathrm{M}$ $\mathrm{EuCl}_{3} . \mathrm{xH}_{2} \mathrm{O}$ solution in water. The other solution contained $92 \mathrm{~g}$ of $0.1 \mathrm{M} \mathrm{Na}_{2} \mathrm{~S}$ as the aqueous part. The two solutions were mixed, and a white suspension was formed. After centrifugation, the particles were rinsed with water and acetone and dried in a vacuum.

Heat treatments of samples were performed to study if $\mathrm{RE}^{3+}$ ions can be incorporated at higher temperatures. Some samples were heated under increased pressure in a Teflon-lined autoclave for $15 \mathrm{~h}$ at $120^{\circ} \mathrm{C}$ (hydrothermal treatment) before the centrifuging step. Precipitated nanocrystalline ZnS/Eu samples made with twice the amount of $\mathrm{Na}\left(\mathrm{PO}_{3}\right)_{n}(20.4 \mathrm{~g})$ were subjected to a heat treatment. Part of the samples were placed in an aluminum oxide crucible, covered by a layer of sulfur, and placed in the oven. First, the samples were heated at 200 ${ }^{\circ} \mathrm{C}$ for $2 \mathrm{~h}$ in a nitrogen atmosphere to evaporate any water present in the sample or the oven tube. After this heat treatment, the samples were heated for another $3 \mathrm{~h}$ at 200, 400,600 , and $800^{\circ} \mathrm{C}$, respectively, in a nitrogen atmosphere. After cooling, the samples were washed several times with distilled water and ethanol to remove any excess sulfur. Finally, the samples were dried in a vacuum.

2.2. Synthesis of Nanocrystalline ZnS/T $\mathbf{b}^{3+}$. Two synthesis routes were used to synthesize nanocrystalline ZnS/ $\mathrm{Tb}^{3+}$ : a conventional preci pitation method and an organometallic method. The first method resembles the standard precipitation method described in section 2.1. A solution of 0.01 $\mathrm{M} \mathrm{TbCl}{ }_{3} \cdot 6 \mathrm{H}_{2} \mathrm{O}$ in water was used as the terbium precursor solution. Sometimes, LiF was added as charge compensator. In addition to this synthesis in water, the synthesis was performed in methanol.

The organometallic method used is similar to the method described by Bhargava et al. ${ }^{22}$ In a nitrogen atmosphere (glovebox), $2.5 \mathrm{~mL}$ of a $1.1 \mathrm{M} \mathrm{Zn}\left(\mathrm{C}_{2} \mathrm{H}_{5}\right)_{2}$ solution in toluene (Aldrich) was further diluted to $205 \mathrm{~mL}$. To this solution, 5 $\mathrm{mL}$ of a $0.011 \mathrm{M}$ solution of terbium trifluoromethanesulfonate (Aldrich) in tetrahydrofuran and $1.1 \mathrm{~mL}$ methacrylic acid (stabilizer, Aldrich) were added. After the addition of $50 \mathrm{~mL}$ of a saturated solution of $\mathrm{H}_{2} \mathrm{~S}$ in toluene $(0.631 \mathrm{M})$, a white suspension was formed instantaneously. The particles were separated from the solution by centrifugation. The separated particles were rinsed with tetrahydrofuran and methanol and dried in a vacuum.

2.3. Nanocrystalline ZnS/E r. Samples of nanocrystalline $\mathrm{ZnS} / \mathrm{Er}$ were prepared by ion implantation at the FOM institute for Atomic and Molecular Physics in Amsterdam. Prior to ion implantation, undoped nanocrystalline ZnS was prepared using the precipitation method described in section 2.1. This undoped sample was implanted with $100 \mathrm{keV}$ Er to a fluence of $1 \times 10^{15} \mathrm{Er}$ ions per $\mathrm{cm}^{2}$. This corresponds to approximately $10 \mathrm{Er}$ ions per nanoparticle. The sample was subsequently annealed at $150-800{ }^{\circ} \mathrm{C}$ for various times between $10 \mathrm{~s}$ to $20 \mathrm{~min}$.

2.4. Characterization. Emission and excitation spectra were recorded with a SPEX Fluorolog spectrofluorometer equipped with two double grating 0.22 m SPEX 1680 monochromators and a $450 \mathrm{~W}$ xenon lamp as excitation source. The emission was detected with a cooled Hamamatsu R928 photomultiplier. Emission spectra were corrected for the sensitivity of the photomultiplier tube, and excitation spectra were corrected for the intensity of the xenon lamp.

$X$-ray powder diffraction patterns were recorded using a Philips PW 1729 X-ray generator with $\mathrm{Cu} K \alpha$ radiation $(\lambda=$ $1.542 \AA$ ). From the line broadening of the XRD patterns, the average particle size was calculated. ${ }^{38}$

\section{Results and Discussion}

3.1. Nanocrystalline ZnS/E $u$ and CdS/E u. X-ray powder diffraction patterns of the ZnS/E u nanoparticles

(38) Cullity, B. D. Elements of X-ray Diffraction; Addison-Wesley: Massachusetts, 1978; p 102.

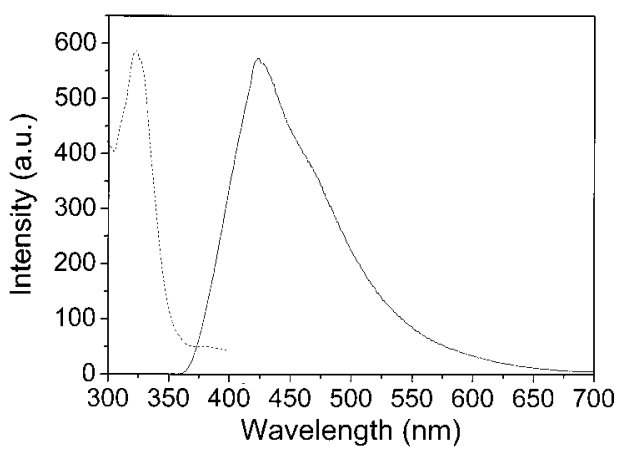

Figure 1. Emission ( $\lambda_{\text {exc }}=330 \mathrm{~nm}$ ) (solid line) and excitation spectra $\left(\lambda_{\mathrm{em}}=480 \mathrm{~nm}\right.$ ) (dashed line) of nanocrystalline ZnS/ $\mathrm{Eu}$, prepared by using the precipitation method, measured at room temperature.

show broad lines at positions that are in agreement with the zincblende modification of $\mathrm{ZnS}$. From the line broadening of the XRD patterns, the average particle size of the samples was cal culated. ${ }^{34}$ When the samples prepared with twice the amount of PP in a nitrogen atmosphere were heated, the particle size increased drastically. Before they were heated, the particle diameter was $5.0 \mathrm{~nm}$. After the samples were heated at 200 ${ }^{\circ} \mathrm{C}$ for $5 \mathrm{~h}$, the average particle diameter increased to $10.2 \mathrm{~nm}$. The heating above $200{ }^{\circ} \mathrm{C}$ (at 400, 600, and $800^{\circ} \mathrm{C}$ ) yiel ded an average particle diameter larger than $20 \mathrm{~nm}$. Due to this large particle size, quantum size effects are not expected for these samples. As a result of the hydrothermal treatment $\left(15 \mathrm{~h}\right.$ at $120^{\circ} \mathrm{C}$ in Teflonlined autoclave), the average particle diameter of the precipitated samples increases from 4.4 to $5.2 \mathrm{~nm}$. By subjection of the samples, made by the microemulsion method, to the hydrothermal treatment $\left(15 \mathrm{~h}\right.$ at $120^{\circ} \mathrm{C}$ in Teflon-lined autoclave), the average particle diameter increased from 4.2 to $7.8 \mathrm{~nm}$.

Similar trends were found for nanocrystalline CdS/ Eu. The average particle diameter of samples prepared with the precipitation method was $3.6 \mathrm{~nm}$, while samples prepared with the microemulsion method had an average particle diameter of $4.6 \mathrm{~nm}$. Due to the hydrothermal treatment ( $15 \mathrm{~h}$ at $120^{\circ} \mathrm{C}$ in Teflon-lined autoclave), the average particle radius of the latter samples increased to $9.6 \mathrm{~nm}$.

A typical emission and excitation spectrum of nanocrystalline ZnS/Eu prepared with the precipitation method is shown in Figure 1. A broad emission band was obtained for the $330 \mathrm{~nm}$ excitation. No sharp $\mathrm{Eu}^{3+}$ emissions or a broad $\mathrm{Eu}^{2+}$ emission around $530 \mathrm{~nm}$ were observed for host lattice excitation, contrary to other publications.9,19 The emission and excitation spectra are similar to the emission and excitation spectrum of undoped nanocrystalline $\mathrm{ZnS}$, showing that no $\mathrm{Eu}^{3+}$ or $\mathrm{Eu}^{2+}$ was incorporated. Also, if $\mathrm{LiCl}$ was used as charge compensator, $\mathrm{Eu}^{3+}$ or $\mathrm{Eu}^{2+}$ emission was not observed. For excitation at $395 \mathrm{~nm}$ (in the ${ }^{7} \mathrm{~F}_{0} \rightarrow{ }^{5} \mathrm{~L}_{6}$ transition of $\mathrm{Eu}^{3+}$ ), weak ${ }^{5} \mathrm{D}_{0} \rightarrow{ }^{7} \mathrm{~F}_{\mathrm{J}}$ emission could be observed. There was a clear relation between the thoroughness of the washing procedure and the intensity of the $\mathrm{Eu}^{3+}$ emission: if more washing steps were included, the Eu ${ }^{3+}$ emission intensity decreased, supporting the conclusion that the $\mathrm{Eu}^{3+}$ emission results from $\mathrm{Eu}^{3+}$ ions absorbed at the surface.

Samples prepared with the microemulsion method show $\mathrm{Eu}^{3+}$ emission, as is demonstrated in Figure 2. In 

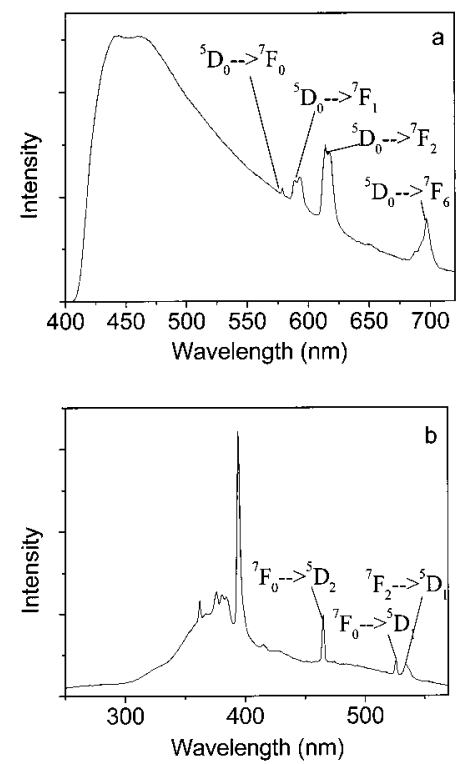

Figure 2. (a) Emission $\left(\lambda_{\mathrm{exc}}=375 \mathrm{~nm}\right)$ and (b) excitation spectra $\left(\lambda_{\mathrm{em}}=612 \mathrm{~nm}\right)$ of nanocrystalline $\mathrm{ZnS} / \mathrm{Eu}$, prepared by using the microemulsion method, measured at room temperature.

the emission spectrum, the characteristic ${ }^{5} \mathrm{D}_{0} \rightarrow{ }^{7} \mathrm{~F}_{\text {J }}$ emission lines of the $\mathrm{Eu}^{3+}$ ion are observed. The spectrum is similar to emission spectra reported for nanocrystalline $\mathrm{ZnS} / \mathrm{Eu}^{3+}$. The observation of $\mathrm{Eu}^{3+}$ emission is, however, no evidence that the $\mathrm{Eu}^{3+}$ ions are incorporated in the ZnS nanocrystals. There may very well be a second phase containing $\mathrm{Eu}^{3+}$, or the (luminescent) $\mathrm{Eu}^{3+}$ ions may be situated at the surface of the nanoparticles. If the $\mathrm{Eu}^{3+}$ ions are indeed incorporated in the ZnS nanocrystals, it is expected that upon excitation of the ZnS host, efficient energy transfer to the $\mathrm{Eu}^{3+}$ centers will occur, similar to the situations for $\mathrm{Mn}^{2+}$ in nanocrystalline $\mathrm{ZnS} / \mathrm{Mn}^{2+}$ and RE ions in bulk ZnS. ${ }^{5-7,26-28}$ A crucial test for the incorporation is an excitation spectrum of the $\mathrm{Eu}^{3+}$ emission; if the $\mathrm{ZnS}$ absorption band is present in the excitation spectrum, energy transfer from the ZnS semiconductor nanoparticle to the $\mathrm{Eu}^{3+}$ ion occurs and it is cl ear that $\mathrm{Eu}^{3+}$ ions are incorporated in the ZnS nanoparticles. If the excitation spectrum does not show the ZnS absorption band, it can be concluded that the $\mathrm{Eu}^{3+}$ emission does not originate from $\mathrm{Eu}^{3+}$ in ZnS nanoparticles. In Figure 2, the excitation spectrum of the $\mathrm{Eu}^{3+}$ emission is also shown. The spectrum is dominated by intraconfigurational $4 \mathrm{f}^{\mathrm{n}} \rightarrow 4 \mathrm{f}^{\mathrm{n}}$ transitions, the strongest line being the ${ }^{7} \mathrm{~F}_{0} \rightarrow{ }^{5} \mathrm{~L}_{6}$ line around $395 \mathrm{~nm}$ as is usually observed for $\mathrm{Eu}^{3+}$. The $\mathrm{ZnS}$ absorption band is not present in the excitation spectrum, showing that the $\mathrm{Eu}^{3+}$ ion cannot be excited via the $\mathrm{ZnS}$ host. This shows that the $\mathrm{Eu}^{3+}$ ions are not incorporated in the ZnS nanoparticles.

The emission spectra for $\mathrm{Eu}^{3+}$ ions adsorbed at the surface of nanocrystalline $\mathrm{ZnS}^{25}$ and nanocrystalline $\mathrm{CdS}$ adsorbed on $\mathrm{Eu}^{3+}$-doped silica gel or zirconia films ${ }^{30,31}$ are similar to the spectra reported here for nanocrystalline ZnS/E u. Probably, the $\mathrm{Eu}^{3+}$ ions in the present compounds are located at the surface.

In the literature, the papers on $\mathrm{ZnS} / \mathrm{RE}^{3+}$ often show only emission spectra, e.g., refs 9 and 29, which makes it difficult to verify if the rare earth ions are incorporated. If excitation spectra are shown, e.g., ref 17 , only

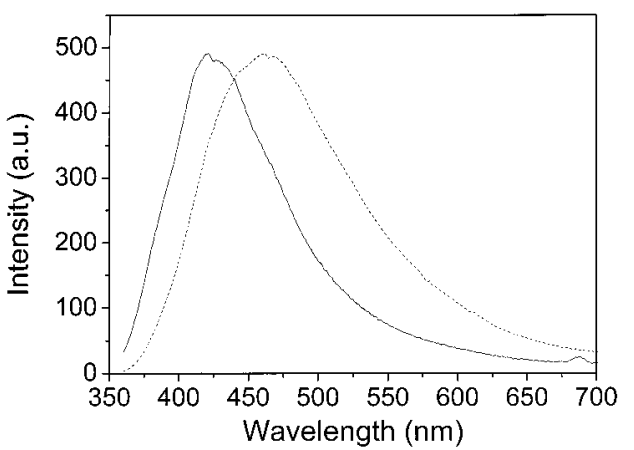

Figure 3. Emission spectrum ( $\lambda_{\text {exc }}=330 \mathrm{~nm}$ ) of nanocrystalline $\mathrm{ZnS} / \mathrm{Eu}$, prepared by using the precipitation method with (dashed line) and without (solid line) hydrothermal treatment, measured at room temperature.

intraconfigurational $4 \mathrm{f}^{\mathrm{n}} \rightarrow 4 \mathrm{f}^{\mathrm{n}}$ excitation lines are present and there are no host lattice (ZnS) excitation bands showing that the $\mathrm{RE}^{3+}$ ions are not incorporated in the nanoparticles, even though it is suggested that they are in the literature.

There are several reasons for the poor incorporation of $\mathrm{Eu}^{3+}$ in nanocrystalline $\mathrm{ZnS}$. First, the ionic radius of the $\mathrm{Eu}^{3+}$ ion is larger than that of $\mathrm{Zn}^{2+}\left(\mathrm{Eu}^{3+}=0.95\right.$ and $\mathrm{Zn}^{2+}=0.75 \AA^{23}$ ). For an $\mathrm{Eu}^{3+}$ ion on a $\mathrm{Zn}^{2+}$ lattice site, the ZnS host lattice has to deform, which is energetically unfavorable. Besides, due to thelarge ionic radius, the $\mathrm{Eu}^{3+}$ ion prefers sites with high coordination numbers (six or higher). In ZnS, however, the coordination number of the cation lattice site is only four, which is very unusual for $\mathrm{Eu}^{3+}$. In addition, the $3+$ charge of the $\mathrm{Eu}^{3+}$ ion has to be compensated for somewhere in the lattice. It is questionable if Eu can be incorporated in a sulfide in the trivalent state. The divalent state is expected to be more stable. ${ }^{39}$

It is known that RE ions can be incorporated in bulk II-VI semiconductors. ${ }^{25}$ However, high temperatures $\left(\sim 900-1200{ }^{\circ} \mathrm{C}^{25}\right)$ are needed to accomplish this. The synthesis conditions used here and in other publications for the preparation of $\mathrm{Eu}^{3+}$-doped nanocrystalline semiconductors are very mild compared to the conditions used for bulk $\mathrm{Eu}^{3+}$-doped semiconductors.

To try and incorporate $\mathrm{Eu}^{3+}$ ions into the nanocrystals, some of the samples were subjected to a heat treatment (up to $800{ }^{\circ} \mathrm{C}$ for $3 \mathrm{~h}$ ) or a hydrothermal treatment ( $15 \mathrm{~h}$ at $120^{\circ} \mathrm{C}$ in a Teflon-lined autoclave). When the samples were heated, the particle size increased dramatically (>10 nm). The samples subjected to both heat and hydrothermal treatment give emission and excitation spectra similar to the ones shown in Figure 2. Upon host lattice excitation at $320 \mathrm{~nm}$, no $\mathrm{Eu}^{3+}$ emission was observed. The hydrothermally treated samples, however, show an increase in luminescence intensity at an emission wavelength around $500 \mathrm{~nm}$ for host lattice excitation (see Figure 3 ). This could possibly suggest that not $\mathrm{Eu}^{3+}$ but $\mathrm{Eu}^{2+}$ is incorporated in the ZnS nanoparticles. It is known that Eu in sulfides is often divalent. ${ }^{39}$ Besides, Chen et al. ${ }^{19}$ reported that $\mathrm{Eu}^{2+}$ could be incorporated in nanocrystalline $\mathrm{ZnS}$ by using a precipitation method, followed by a heat treatment at $80{ }^{\circ} \mathrm{C}$ for $24 \mathrm{~h}$. Upon excitation in the nano-

(39) Blasse, G.; Grabmaier, B. C. Luminescent Materials; SpringerVerlag; Berlin 1994. 

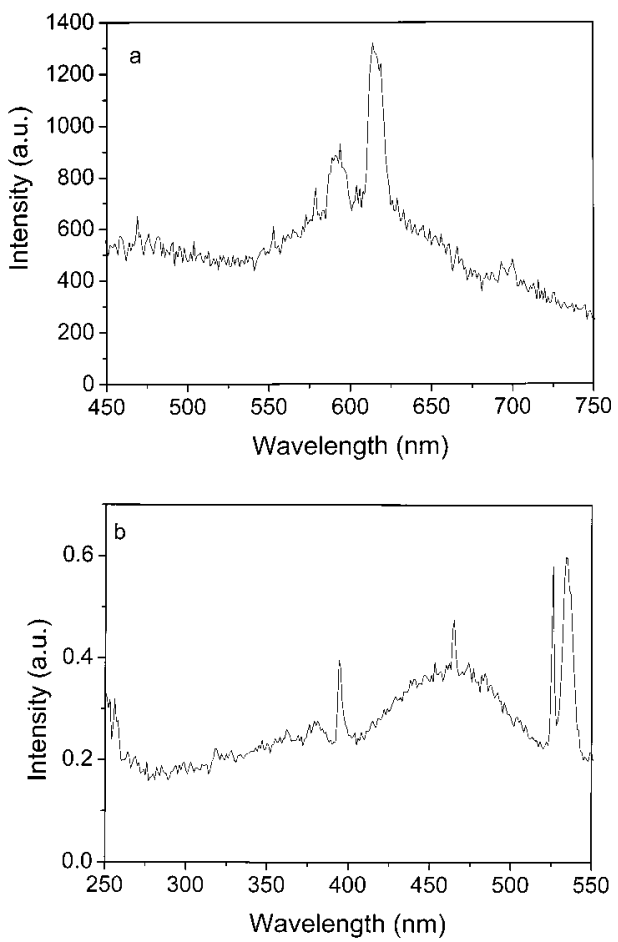

Figure 4. (a) Emission ( $\lambda_{\text {exc }}=380 \mathrm{~nm}$ ) and (b) excitation spectra $\left(\lambda_{\mathrm{em}}=620 \mathrm{~nm}\right)$ of nanocrystalline CdS/Eu, prepared by using the microemulsi on method, measured at room temperature.

crystalline host, an emission at around $530 \mathrm{~nm}$ was obtained, which was assigned to $\mathrm{Eu}^{2+}$ emission. $\mathrm{Xu}^{9}$ reported a similar emission for ZnS/Eu prepared by a microemulsion method, followed by a hydrothermal treatment. They assigned this emission to the photoionization of $\mathrm{Eu}^{2+}$ to $\mathrm{Eu}^{3+}$. Neither of these publications verified the absence of this emission band in undoped nanocrystalline ZnS, prepared in the same way as the doped samples. It is possible that due to the applied heat or the hydrothermal treatments, new luminescent centers are formed in the nanocrystalline host, which cause the observed broad green emission.

To investigate the influence of the hydrothermal treatment on the luminescence of undoped nanocrystalline ZnS, an undoped nanocrystalline ZnS sample, made by using the microemulsion method, was subjected to the hydrothermal treatment. The same increase of the emission spectrum around $500 \mathrm{~nm}$ was observed for the undoped sample as for the Eu-doped one. Thus, upon subjecting ZnS nanoparticles to heat or hydrothermal treatment, new luminescence centers are created.

Since the ionic radius of the $\mathrm{Cd}$ ion is larger than the ionic radius of the $\mathrm{Zn}^{2+}$ ion $(0.98$ and $0.74 \AA$, respectively ${ }^{23}$ ), it is expected that $\mathrm{Eu}^{2+}$ or $\mathrm{Eu}^{3+}$ is incorporated more easily in CdS than in ZnS. As for nanocrystalline $\mathrm{ZnS} / \mathrm{Eu}$, the preci pitated samples gave only CdS defect emission around $650 \mathrm{~nm}$ for host lattice excitation (see Figure 4). The samples prepared with the microemulsion method gave $\mathrm{Eu}^{3+}$ emissions, but as was observed for nanocrystalline ZnS/E u, these emissions were relatively weak and could be most efficiently excited internally in the $\mathrm{Eu}^{3+}$, as was al so reported by Morita et al ${ }^{20}$ showing that the $\mathrm{Eu}^{3+}$ ions are not incorporated in the nanocrystalline $\mathrm{CdS} / \mathrm{Eu}$. Also, the addition of $\mathrm{LiCl}$ as charge compensator or after the subjection of the samples to a hydrothermal treatment, no $\mathrm{Eu}^{3+}$ was incorporated in the nanocrystalline CdS. Again, the lowenergy side of the defect emission increased upon hydrothermal treatment of nanocrystalline CdS/Eu. This increase was also observed for undoped nanocrystalline CdS. This low-energy emission is therefore not ascribed to $\mathrm{Eu}^{2+}$ emission but to the emission from newly created luminescent centers.

From the above discussion, we conclude that by the use of a common precipitation method or a microemulsion method to synthesize nanocrystalline ZnS/Eu or $\mathrm{CdS} / \mathrm{Eu}$, the $\mathrm{Eu}^{3+}$ or $\mathrm{Eu}^{2+}$ ions are not incorporated in ZnS or CdS host. The characteristic ${ }^{5} D_{0} \rightarrow{ }^{7} F_{j}$ emission that is observed results from $\mathrm{Eu}^{3+}$ ions adsorbed at the surface of the nanocrystalline ZnS or CdS particles or a small amount of a second phase.

3.2. Nanocrystalline ZnS/T $\mathbf{b}^{3+}$. The first publication on nanocrystalline ZnS/Tb ${ }^{3+}$ appeared in $1994 .{ }^{40}$ Bhargava reported that upon excitation in the nanocrystalline ZnS host, the well-known ${ }^{5} \mathrm{D}_{4}-{ }^{7} \mathrm{~F}_{\mathrm{n}}$ emissions of the $\mathrm{Tb}^{3+}$ ion around $550 \mathrm{~nm}$ were observed. Later, it was reported by the same author ${ }^{15}$ that the lifetime of the $\mathrm{Tb}^{3+}$ emission was shortened from $\sim 3 \mathrm{~ms}$ for bulk $\mathrm{ZnS} /$ $\mathrm{Tb}^{3+}$ to $7 \mathrm{~ns}$ in nanocrystalline $\mathrm{ZnS} / \mathrm{Tb}^{3+}$. This lifetime shortening was explained by the quantum size effects. ${ }^{15}$

A similar explanation was given for the observed lifetime shortening of the $\mathrm{Mn}^{2+}$ emission of nanocrystalline $\mathrm{ZnS} / \mathrm{Mnn}^{2+} .{ }^{5}$ However, recently, the presence of lifetime shortening in nanocrystalline $\mathrm{ZnS} / \mathrm{Mn}^{2+}$ was shown not to occur. ${ }^{6,7}$ The $\mathrm{Mn}^{2+}$ emission in nanocrystalline $\mathrm{ZnS} / \mathrm{Mn}^{2+}$ has a "normal" millisecond lifetime, like in bulk $\mathrm{ZnS} / \mathrm{Mn}^{2+}$. Therefore, lifetime shortening in $\mathrm{ZnS} / \mathrm{Tb}^{3+}$ is expected to be due to the same misinterpretation.

Ihara et al. ${ }^{17}$ observed ${ }^{5} D_{4}-{ }^{7} F_{n}$ emissions for nanocrystalline $\mathrm{ZnS} / \mathrm{Tb}^{3+}$ as well. In addition, they observed a lifetime shortening from $1.15 \mathrm{~ms}$ for bulk $\mathrm{ZnS} / \mathrm{Tb}^{3+}$ to $0.10 \mathrm{~ms}$ for nanocrystalline $\mathrm{ZnS} / \mathrm{Tb}^{3+}$. However, close inspection of the excitation spectra of these emissions reveals that the $\mathrm{Tb}^{3+}$ emission can only be excited via $4 \mathrm{f}^{\mathrm{n}} \rightarrow 4 \mathrm{f}^{\mathrm{n}}$ excitation of the $\mathrm{Tb}^{3+}$ ion and via $\mathrm{ZnS}$ band gap excitation, showing that the $\mathrm{Tb}^{3+}$ ions are not incorporated in the ZnS host lattice.

Kane and co-workers ${ }^{16}$ tried to incorporate $\mathrm{Tb}^{3+}$ in nanocrystalline ZnS using block copolymer nanoreactors. Emissions characteristic for $\mathrm{Tb}^{3+}$ were observed. However, an excitation spectrum of these emissions was not shown in this paper. It is therefore not clear if the $\mathrm{Tb}^{3+}$ ions are incorporated in the $\mathrm{ZnS}$ host lattice.

To incorporate $\mathrm{Tb}^{3+}$ ions in a nanocrystalline $\mathrm{ZnS}$ host lattice, a common precipitation method was used. F urthermore, we applied the organometallic synthesis method used by Bhargava and co-workers. The samples prepared with the common precipitation method gave a broad emission band around $430 \mathrm{~nm}$, like in Figure 1 , upon host lattice excitation. $\mathrm{No} \mathrm{Tb}^{3+}$ emission was observed. The addition of LiF as charge compensator did not yield $\mathrm{Tb}^{3+}$ emission. The synthesis was also performed in methanol. Since $\mathrm{TbCl}_{3}$ is less soluble in methanol than in water, it was expected that $\mathrm{Tb}^{3+}$ would incorporate more easily in the ZnS if the synthe-

(40) Bhargava, R. N.; Gallagher, D.; Welker, T. J . Lumin. 1994 60-61, 275. 

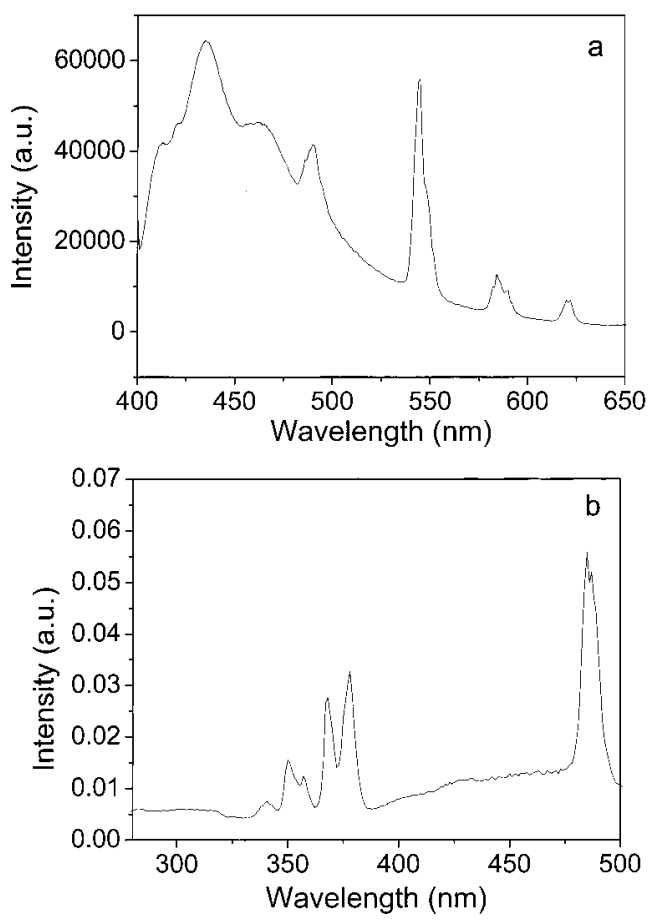

Figure 5. (a) Emission ( $\lambda_{\text {exc }}=378 \mathrm{~nm}$ ) and (b) excitation spectra $\left(\lambda_{\mathrm{em}}=544 \mathrm{~nm}\right)$ of nanocrystalline $\mathrm{ZnS} / \mathrm{Tb}^{3+}$, prepared by using the organometallic method, measured at room temperature.

sis was done in methanol. However, no $\mathrm{Tb}^{3+}$ emission lines were observed for host lattice excitation.

This was tried to reproduce the organometallic synthesis that Bhargava used to prepare nanocrystalline $\mathrm{ZnS} / \mathrm{Tb}^{3+}$. Since a detailed description of the synthesis route was never published, it was assumed that an organometallic synthesis method used by the same authors, comparable to the organometallic synthesis method used for the preparation of nanocrystalline ZnS/ $\mathrm{Mn}^{2+}$, was used to prepare nanocrystalline $\mathrm{ZnS} / \mathrm{Tb}^{3+}$.

Again, no $\mathrm{Tb}^{3+}$ emission was observed for host lattice excitation of these samples. Only if the $\mathrm{Tb}^{3+}$ ions were excited in characteristic $4 \mathrm{f}^{\mathrm{n}} \rightarrow 4 \mathrm{f}^{\mathrm{n}}$ lines, ${ }^{5} \mathrm{D}_{4}-{ }^{7} F_{\mathrm{n}}$ emissions of the $\mathrm{Tb}^{3+}$ ion could be observed (see Figure 5). From this, it is concluded that with the application of room-temperature preparation techniques, $\mathrm{Tb}^{3+}$ cannot be incorporated in nanocrystalline $\mathrm{ZnS}$. As was discussed in section 3.1, this is due to the large size, chemical differences, and the need for charge compensation.
3.3. Nanocrystalline ZnS/Er. From the above discussion, it is clear that incorporation of rare earth ions in nanocrystal line semiconductors by several chemical preparation methods was not successful. Therefore, ion implantation was used to incorporate $\mathrm{Er}$ in nanocrystalline $\mathrm{ZnS} . \mathrm{Er}^{3+}$ in bulk ZnS shows a green emission originating from the ${ }^{4} \mathrm{~S}_{3 / 2} \rightarrow{ }^{4} \mathrm{I}_{15 / 2}$ transition of the $\mathrm{Er}^{3+}$ ion. ${ }^{24}$ After ion implantation, the samples were thermally annealed, since ion implantation damages the crystal structure of the sample, which causes luminescence quenching. Thermal annealing also increases the particle size of the semiconductors. Thermal annealing at $450{ }^{\circ} \mathrm{C}$ for $10 \mathrm{~min}$ yielded samples with an average particle diameter of $5.6 \mathrm{~nm}$, while before thermal annealing, the average particle size was $4.2 \mathrm{~nm}$. The samples annealed at this temperature did not show $\mathrm{Er}$ luminescence. Only the defect-related ZnS emission was observed. Possibly, the crystal structure around the Er ions is not restored by thermal annealing at this temperature. Therefore, another sample was subjected to $800{ }^{\circ} \mathrm{C}$ for $10 \mathrm{~min}$. Still, no $\mathrm{Er}$ luminescence was found for this sample. Due to this high temperature, the particle diameter increases to above $20 \mathrm{~nm}$. Hence, initial ion implantation experiments of precipitated ZnS samples followed by thermal annealing have not been successful in the preparation of nanocrystalline II-VI semiconductors doped with RE ions.

\section{Conclusion}

Nanocrystalline II-VI semiconductor (ZnS and CdS) particles were prepared in the presence of trivalent rare earth dopant ions $\left(\mathrm{Eu}^{3+}\right.$ and $\left.\mathrm{Tb}^{3+}\right)$. Several synthesis methods were used based on commonly applied precipitation methods and microemulsion techniques. Luminescence measurements show that the rare earth ions are not incorporated in the semiconductor nanoparticles. Even after the samples are heated to $800{ }^{\circ} \mathrm{C}$ (resulting in a substantial increase in particle size), there is no evidence that the trivalent lanthanides are inside the II-VI semiconductor nanoparticles.

Acknowledgment. Prof. Albert Polman is gratefully acknowledged for making it possible for us to use the ion implantation setup at the FOM Institute of Atomic and Molecular Physics in Amsterdam, and Pieter Kik and Freek Suyver are acknowledged for their hel $p$ with the ion implantation.

CM011195S 